\title{
Probing the surfaces of core-shell and hollow nanoparticles by solvent relaxation NMR
}

\author{
M.R. Hossain*, D. Wray*, A. Paul ${ }^{\%}$ and P.C. Griffiths* \\ * Department of Pharmaceutical, Chemical and Environmental Science, University of Greenwich, \\ Central Avenue, Chatham Maritime, Kent ME4 4TB \\ ${ }^{\%}$ School of Chemistry, Cardiff University, Main Building, Park Place, Cardiff CF10 3TB
}

\begin{abstract}
Measurement of the spin-spin NMR relaxation time (or its inverse, the rate) of water molecules in aqueous nanoparticle dispersions has become a popular approach to probe of the nature and structure of the particle surface and any adsorbed species. Here, we report on the characterisation of aqueous dispersions of hollow amorphous nanoparticles, that have two liquid accessible surfaces (inner cavity surface and outer shell surface), plus the solid (silica) and core-shell (titania-silica) nanoparticle precursors from which the hollow particles have been prepared. In all cases, the observed water relaxation rates scale linearly with particle surface area, with the effect being more pronounced with increasing levels of titania present at the particle surface. Two distinct behaviours were observed for the hollow nanoparticles at very low volume fractions, which appear to merge with increasing surface area (particle concentration). Herewith, we further show the versatility of solvent NMR spectroscopy as a probe of surface character.
\end{abstract}




\section{Introduction}

Recent years have witnessed the explosion of the application of nanotechnology to medicine, specifically nanoparticles for targeted drug delivery [1] [2] [3] [4]. These formulations often present multiple challenges during their development, that require a thorough understanding of the formulation at the molecular level. In such a nanoparticulate formulation, nanoparticles are usually dispersed in a liquid system at high volume fraction, and therefore, it is important control the stability of such dispersions, this being sensitive to the total surface area available and its interaction with the formulation components, e.g. polymers, surfactants.

NMR constitutes a family of experimental methodologies, that provides structural and dynamical insights from the nano- to the macroscale. Early work in the area of heterogeneous or hierarchal systems focused on porous materials, with the effects of the surface/pore modulating the relaxation time of the adsorbed species [5] [6]. Here, determination of the relaxation time of the solvent (socalled "solvent relaxation NMR") has been used to analyze three nanoparticulate dispersions, namely(i) solid silica nanoparticles with a pure silica surface, (ii) solid silica-titania core-shell nanoparticles (STCSNP) grown from the seed silica, that possess a pure titania surface, and (iii) hollow silica-titania nanoparticles (STHNP) with a mixed silica-titania surface.

NMR relaxation of the solvent has evolved into a powerful and non-invasive characterisation technique, in which the average mobility of the solvent - reflected in its spin-spin relaxation time $\mathrm{R}_{2}$ is analysed to extract information on the surface by virtue of its impact on the dynamics of the solvent molecules whilst in the vicinity of the surface [7] [8] [9] [10].

In a relaxation measurement, water molecules in a particulate dispersion undergo fast exchange between a motionally restricted and/or anisotropic state in close proximity to the surface of the particle and the non-restricted, isotropic state in solution. The relaxation rates in these two environments are quite different due to the highly constrained mobility of the water molecule near the surface and the presence of nuclei of different magnetic behaviour, i.e. the surface composition [8] Typically, the proton in the motionally restricted state undergoes much faster relaxation $\left(\mathrm{R}_{2 \text { restricted }}\right)$ than the proton in the isotropic state, colloquially termed "free" $\left(\mathrm{R}_{2 \mathrm{free}}\right)$. As long as fast exchange occurs between two environments, the total relaxation rate of the system is the average relaxation rate weighted by the time the proton spends in each of the two environments.

Thus, the single relaxation rate, $\mathrm{R}_{2}$, for such a fast exchange limit is calculated from the following equation: 
where, $p_{\text {restricted }}$ is the time-averaged probability that a given proton/water molecule experiences motional restriction due to its proximity to the particle surface. The specific relaxation rate, $\mathrm{R}_{2 \mathrm{SP}} v i z$ $\mathrm{R}_{2}$ normalized to the relaxation rate of the background solvent $\left(\mathrm{R}_{2}{ }^{\circ}\right)$ is usually presented to minimise the effects of subtle changes in solvent and temperature [7];

$$
R_{2 S P}=\frac{R_{2}}{R_{2}^{\circ}}-1
$$

In the case of bare particles of a given surface composition, an increase in $\mathrm{R}_{2 \mathrm{SP}}$ means that there is a greater level of motionally restricted water (i.e. more efficient relaxation behaviour of water nuclei). As a tool to examine polymer adsorption, an enhancement in $\mathrm{R}_{2 \mathrm{SP}}$ is observed as more water becomes trapped at the polymer-particle interface, thereby decreasing the mobility of the water molecule, and/or increasing their retention time at the interface [11].

\section{Experimental Section}

\section{Materials}

Particles: silica, silica-titania core-shell (STCSNP) and silica-titania hollow nanoparticles (STHNP) used here were synthesized following published procedures [4], [12]. Briefly, silica nanoparticles were prepared from tetraethyl orthosilicate through the sol-gel process in the presence of ammonium hydroxide. Silica-titania core-shell nanoparticles (STCSNP) were subsequently prepared from these precursor silica nanoparticles through the sol-gel reaction of titanium isopropoxide (TTIP) onto the silica surface. Finally, silica-titania hollow nanoparticles (STHNP) were synthesized upon sonication of the core-shell particles in presence of dilute ammonium hydroxide solution [12]. All samples were filtered through glass wool and dialyzed for 3 days against deionized water $\left(18 \mathrm{M} \Omega \mathrm{cm}^{-1}\right)$ before NMR measurement. All dispersions were visually stable over several weeks, with no change in particle size. The size, figure 1, and surface areas of three different structured nanoparticles are listed in Table 1.

\section{Particle characterisation}

The particles were characterised by a combination of standard techniques; dynamic light scattering (Malvern Nano ZS) to quantify particle size (figure 1); X-ray powder diffraction (Bruker D8 Advance 
$\mathrm{X}$-ray diffractometer) to assess the presence of crystallinity; scanning transmission electron microscopy (Hitachi SU8030) to determine core and shell dimensions; nitrogen adsorption (Micromeritics 3FLEX) to probe surface area and pore size distributions; and energy dispersive X-ray spectroscopy (Thermo-Noran NSS system 7) to characterise the atomic composition of the particle/shell.

The absence of any peaks in the X-ray diffraction pattern (data not presented) indicated that the particles were largely amorphous, though it is not possible to discount the presence of small degrees of crystallinity due to the low sizes of these particles. That said, there was no evidence for such in the WAXS data. Further, the TEM images (data not presented) confirmed the respective morphologies, with the inner void of the hollow particle having a dimension of $32( \pm 5) \mathrm{nm}$.

\section{Solvent relaxation NMR}

The NMR spectrometer (Acorn Drop ${ }^{\mathrm{TM}}$ ) used here is a temperature controlled bench-top device supplied by Xigo Nanotools, Inc., Bethlehem, USA. Acorn Drop ${ }^{\mathrm{TM}}$ operates at $13 \mathrm{MHz}$. Pulse lengths of $5.9 \mu \mathrm{s}\left(90^{\circ}\right)$ and $11.2 \mu \mathrm{s}\left(180^{\circ}\right)$ were used for all measurements. The spin-spin relaxation rate of water was measured using the CPMG (Carr-Purcell-Meiboom-Gill) [13] [14] pulse sequence with a typical spacing of $1 \mathrm{~ms}$ between $180^{\circ}$ pulses (i.e., $\tau=0.5 \mathrm{~ms}$ ). The recycle delay of at least $5 T_{l}$ (spinlattice relaxation time) was maintained between 4 consecutive scans for full recovery of magnetization. The spin-spin relaxation rate $\left(R_{2}\right)$ was calculated from each magnetization relaxation decay curve, $M_{y}(t)$, where each curve was fitted against time $t$ to the following equation using a nonlinear least-squares algorithm:

$$
M_{y}(t)=M_{y}(0) e^{-\left(t R_{2}\right)}
$$

where $M_{y}(0)$ denotes the immediate transverse magnetisation after $90^{\circ}$ pulse sequence.

For hollow nanoparticles, where two surfaces are exposed to the liquid medium, the measured NMR signal might be expected to be the sum of the signal of water associated with the inner surface of the hollow cavity, the outer surface of the particle and the signal from free water in bulk. If transfer between the inner and outer volumes is not rapid compared to the NMR timescale, i.e. there are two environments, but within these distinct volumes, the water is in fast exchange between the surface and 
the respective non-surface species, one would anticipate that magnetization decays will be double exponential;

$M(t)=M_{\text {outer }} \exp \left(-R_{\text {outer }} t\right)+M_{\text {inner }} \exp \left(-R_{\text {inner }} t\right)$

where $M(t)$ denotes the total signal, $R_{\text {inner }}$ and $R_{\text {outer }}$ are the average relaxation rate for the inner and outer surfaces of hollow nanoparticles respectively and $M_{\text {inner }}$ is the signal from the inner surface and $M_{\text {outer }}$, from the outer surface. If however, the inner and outer environments exchange rapidly, then only a globally averaged relaxation behaviour would be observed.

\section{Result and Discussion}

To understand the relaxation behaviour and surface properties of bare silica, silica-titania core-shell (STCSNP) nanoparticles and silica-titania hollow nanoparticles (STHNP), the transverse relaxation time of the solvent as probe, or more specifically, the reciprocal relaxation rate $\left(\mathrm{R}_{2}\right)$ was measured. Figure 2 shows the normalized CPMG decay curves for the three different colloidal dispersions (silica nanoparticles, STCSNP and STHNP dispersed in water) at a given particle concentration together with the fits from eq 3 . The hollow and core-shell nanoparticles cause the magnetization to decay faster than the silica nanoparticles due to a differing surface interaction - an increased number of- or decreased correlation time for the water molecules associated with the particle surface [7]. Therefore the relaxation enhancement follows the order; silica $>$ STCSNP $>$ STHNP. It is also noted that the CPMG curve of the hollow nanoparticles has an additional distinguishing feature compared with the solid core-shell and silica nanoparticles, in that the single exponential fit did not appropriately describe the experimental data. For clarity, the data for the hollow nanoparticles are presented on the semi-log scale (figure 3), analysed in terms of a single exponential fit (figures 3a and 3c), and in terms of a double exponential fit eq 4 (figures $3 \mathrm{~b}$ and $3 \mathrm{~d}$ ). Therefore it is concluded that two relaxation rates are present, and it would be tempting to conclude that these arise from the water adsorbed at the inner and outer surfaces of the hollow nanoparticles. The fitting parameters for a double exponential fit are listed in Table-2.

Figure 4 shows the respective $\mathrm{R}_{2 \mathrm{~A}}$ and $\mathrm{R}_{2 \mathrm{~B}}$ values based on the best fitting for hollow nanoparticle samples as a function of concentration. Surprisingly, the double exponential fit was only required for hollow nanoparticle dispersions at very low volume fractions. The intensity ratio for these two 
components was found to be constant at low volume fraction, i.e. to not vary with particle volume fraction and therefore total surface area over this dilute volume fraction window (figure 5). At higher volume fractions, the components $\left(\mathrm{R}_{2 \mathrm{~A}}\right.$ and $\mathrm{R}_{2 \mathrm{~B}}$ values) of the double exponential fit appear to coalesce into an effective, single exponential decay (figure 4). This is an unexpected observation, and is most likely associated with the rate of exchange between the water molecules constrained within the pore, adsorbed to the outer surface and the bulk phase, conflated with the amount of surface available for the aqueous phase - too little surface and there is insufficient motional averaging between water in the three environments, and the relaxation data detects two distinct environments. Clearly, this is not fully understood and further work is required to characterize this interesting observation.

Turning our attention to higher volume fraction (concentration) dispersions the observed behaviour is less challenging to interpret. In figure $6, \mathrm{R}_{2 \mathrm{SP}}$ for three different structured nanoparticles are shown as a function of weight percentage of particles in aqueous dispersion, and figure 7 shows the $\mathrm{R}_{2 \mathrm{SP}}$ of three colloidal particle surfaces as a function of the particles surface area. The surface areas for the three different colloidal particles were calculated from the average particle radius (measured from scanning-transmission electron microscopy) and volume fraction. Over the concentration range studied, the linear relationship can be seen for each nanoparticulate system which validates the fast exchange model of eq 1 demonstrating the fast proton exchange between restricted and non-restricted environments [15] [7] [16].

Moreover, in figure 7, the slope of the $\mathrm{R}_{2 \mathrm{SP}} v s$. surface area relationship - the relaxation rate enhancement - is large in the case of core-shell and hollow nanoparticles and greater than that of the bare silica nanoparticles. This indicates the differing nature of the surfaces of the nanoparticle, e.g. hydrophilicity, porosity and chemical composition [7]. Table-1 presents the nitrogen physisorption data of these three different colloidal particles. The surface enhancements - the slopes of plots of relaxation enhancement normalised to surface area, figure 7 - indicate the sensitivity of the technique to surface composition, viz $\mathrm{R}_{2 \mathrm{SP}}{ }^{\mathrm{enh}}=2$ (silica), 5 (core-shell) and 3 (hollow particles). In the case of hollow nanoparticles, this calculation will implicitly include those water molecules adsorbed on both the inner and outer surfaces. The effect of surface composition is evident by a largely linear relationship between $\mathrm{R}_{2 \mathrm{SP}}{ }^{\text {enh }}$ and the degree of Ti incorporation into the particles (assuming the shell of the core-shell nanoparticle is entirely Ti), though the presence of small pores (suggested by nitrogen physisorption data) on the hollow nanoparticle surfaces cannot be discounted.

\section{Conclusion}


The nanoparticle dispersions used here, with comparable size, structure but differing surface chemistry, provide very convenient systems on which the solvent relaxation NMR technique can be further thoroughly tested.

There has been an extensive use of solvent relaxation NMR for studying a variety of colloidal particles in liquids. Here this technique was used to study dispersions of silica nanoparticles, coreshell nanoparticles (STCSNP) and hollow nanoparticles (STHNP). Relaxation rates for these three different nanostructures were very different. The titania surfaces of STCSNP and mixed silica-titania surfaces of STHNP show higher $\mathrm{R}_{2 \mathrm{SP}}$ enhancement compared with the silica surface. This high relaxivity of water on pure titania and silica-titania mixed surfaces was shown to be due to the presence of the quadrupole nuclei of titanium once the higher surface areas of core-shell and hollow nanoparticles has been accounted for. For the hollow nanoparticles, this analysis is based on relaxation rates from the inner cavity and outer shell surface. 


\section{Figures}

Figure 1: Size distributions of silica nanoparticles (squares), silica-titania core-shell nanoparticles (STCSNP) (circles) and silica-titania hollow nanoparticles (STHNP) (triangles).

Figure 2: CPMG decay curves for silica nanoparticles (squares), silica-titania core-shell nanoparticles (STCSNP) (circles) and silica-titania hollow nanoparticles (STHNP) (triangles), at fixed volume fraction of 0.0024 .

Figure 3: Relaxation decay curve for the hollow nanoparticles. The solid line through the experimental data (top panels) is the single exponential fit (left) and double exponential fit (right). The lower panels depict the residual plots - the difference between fit and the data - for the hollow nanoparticles calculated from the single exponential fit (left) and double exponential fit (right).

Figure 4: Variation in $\mathrm{R}_{2}$ components for hollow nanoparticles; (triangles up) fast rate and (triangles down) slow rate as a function of volume fraction. At higher volume fractions, only one rate is observed (circles). The solid line is a linear prediction of $\mathrm{R}_{2}$ on surface area.

Figure 5: Intensity ratio (slow / fast) of the two relaxation components at low volume fraction for hollow particles.

Figure 6: Relaxation rate $\left(\mathrm{R}_{2 \mathrm{SP}}\right)$ of silica nanoparticles (squares), silica-titania core-shell nanoparticles (STCSNP) (circles) and silica-titania hollow nanoparticles (STHNP) (triangles) as a function of particle concentration.

Figure 7: Relaxation rate $\left(\mathrm{R}_{2 \mathrm{SP}}\right)$ for silica nanoparticles (squares), silica-titania core-shell nanoparticles (STCSNP) (circles) and silica-titania hollow nanoparticles (STHNP) (triangles) as a function of particle surface area. The surface area used for three different colloidal particles were calculated from the average particle radius (measured from scanning-transmission electron microscopy) and the volume fraction. 


\section{References}

[1] S. K. Misra, F. Ostadhossein, E. Daza, E. V. Johnson and D. Pan, "Hyperspectral Imaging Offers Visual and Quantitative Evidence of Drug Release from Zwitterionic-PhospholipidNanocarbon When Concurrently Tracked in 3D Intracellular Space," Adv. Funct. Mater, vol. 26, p. 8031-8041, 2016.

[2] D. Ren, "Protein Nanoparticle as a Versatile Drug Delivery System in Nanotechnology," $J$ Nanomed Res, vol. 4, p. 1, 2016.

[3] Y. Yang and C. Yu, "Advances in silica based nanoparticles for targeted cancer therapy.," Nanomedicine, vol. 12, no. 2, p. 317-332, 2016.

[4] X. She, L. Chen, Z. Yi, C. Li, C. He, C. Feng, T. Wang, S. Shigdar, W. Duan and L. Kong, "Tailored Mesoporous Silica Nanoparticles for Controlled Drug Delivery: Platform Fabrication, Targeted Delivery, and Computational Design and Analysis," Mini Rev Med Chem., vol. 16, no. 18, pp. 1-14, 2016.

[5] Yi-Qiao Song, "Magnetic Resonance of Porous Media (MRPM): A perspective" Journal of Magnetic Resonance vol. 229, pp. 12-24, 2013

[6] M.Fleury, "Measurement of interfacial area from NMR time dependent diffusion and relaxation measurements" J. Coll. Int. Sci., vol. 509, pp. 495-501

[7] T. Cosgrove, K. S. Jack, N. Green, T. M. Obey and M. Wood, "NMR Solvent Relaxation Studies on Concentrated Particulate Dispersions," ACS Symposium Series, Supramolecular Structure in Confined Geometries, Chapter 10, vol. 736, pp. 153-168, 1999. 
[8] C. L. Cooper, T. Cosgrove, J. S. v. Duijneveldt, M. Murray and S. W. Prescott, "Colloidal Particles in Competition for Stabilizer: A Solvent Relaxation NMR Study of Polymer Adsorption and Desorption," Langmuir, vol. 28, p. 16588-16595, 2012.

[9] D. Fairhurst, T. Cosgrove and W. S. Prescott, "Relaxation NMR as a tool to study the dispersion and formulation behavior of nanostructured carbon materials," Magn. Reson. Chem. , vol. 54, pp. 521-526, 2016.

[10] T. Cosgrove, C. Roberts, T. Garasanin, R. G. Schmidt and G. V. Gordon, "NMR Spin-Spin Relaxation Studies of Silicate-Filled Low Molecular Weight Poly(dimethylsiloxane)s," Langmuir, vol. 18, pp. 10080-10085, 2002.

[11] B. Cattoz, T. Cosgrove, M. Crossman and S. W. Prescott, "Surfactant-Mediated Desorption of Polymer from the Nanoparticle Interface," Langmuir, vol. 28, p. 2485-2492, 2012.

[12] C. Moonjung, K. Chanhoi, J. Soon, Y. Kyoung, L. Jun and J. Jyongsik, "Synthesis of titania embedded silica hollow nanospheres via sonication mediated etching and re-deposition," Chem. Commun., vol. 47, p. 7092-7094, 2011.

[13] H. Y. Carr and E. M. Purcell, "Effects of Diffusion on Free Precession in Nuclear Magnetic Resonance Experiments," Phys. Rev., vol. 94, pp. 630-643, 1954.

[14] S. Meiboom and D. Gill, "Modified Spin-Echo Method for Measuring Nuclear Relaxation Times," Rev. Sci. Instrum., vol. 29, p. 688-691, 1958.

[15] G. P. van der Beek, M. A. Cohen Stuart and T. Cosgrove, "Polymer Adsorption and Desorption Studies via 1H NMR Relaxation of the Solvent," Langmuir, vol. 7, pp. 321-334, 1991.

[16] A. Nelson, K. S. Jack, T. Cosgrove and D. Kozak, "NMR Solvent Relaxation in Studies of Multicomponent Polymer Adsorption," Langmuir, vol. 18, no. 7, p. 2751, 2002. 


\section{Tables}

\begin{tabular}{|l|l|l|l|}
\hline Particle & Silica NPs & Core-shell NPs & Hollow NPs \\
\hline Surface area $\left(\mathrm{m}^{2} / \mathrm{g}\right)$ & $90 \pm 6$ & $180 \pm 7$ & $295 \pm 7$ \\
\hline Particle size $(\mathrm{nm})$ & $30 \pm 2$ & $50 \pm 2$ & $50 \pm 3$ \\
\hline Atom composition $(\% \mathrm{Si})$ & 100 & $74 \pm 5$ & $52 \pm 4$ \\
\hline
\end{tabular}

Table 1: The size distribution and surface area of silica, silica-titania core-shell and silica-titania hollow nanoparticles. For the core-shell particles, it is assumed that the shell consists of titania and the core silica. 


\begin{tabular}{|c|c|c|c|c|}
\hline Parameters & $\mathbf{R}_{\mathbf{2 A}}(\mathbf{m s})$ & $\mathbf{Y}_{\mathbf{A}} / \%$ & $\mathbf{R}_{\mathbf{2 B}}(\mathbf{m s})$ & $\mathbf{Y}_{\mathbf{B}} / \mathbf{\%}$ \\
\hline $\begin{array}{c}\text { Hollow nanoparticle } \\
\text { dispersion in water }\end{array}$ & $1.3( \pm 0.5) \times 10^{-3}$ & $70 \pm 3$ & $5.0( \pm 0.5) \times 10^{-3}$ & $30 \pm 1$ \\
\hline
\end{tabular}

Table 2: Exemplar $\mathrm{R}_{2}$ relaxation data for dilute silica-titania hollow nanoparticles, at particle concentration $0.24 \mathrm{wt} \%$. $\mathrm{Y}_{\mathrm{A}}$ and $\mathrm{Y}_{\mathrm{B}}$ are the fractions (expressed as \%) on the total intensity with relaxation time $\mathrm{R}_{2 \mathrm{~A}}$ and $\mathrm{R}_{2 \mathrm{~B}}$ respectively. 


\section{Figures}

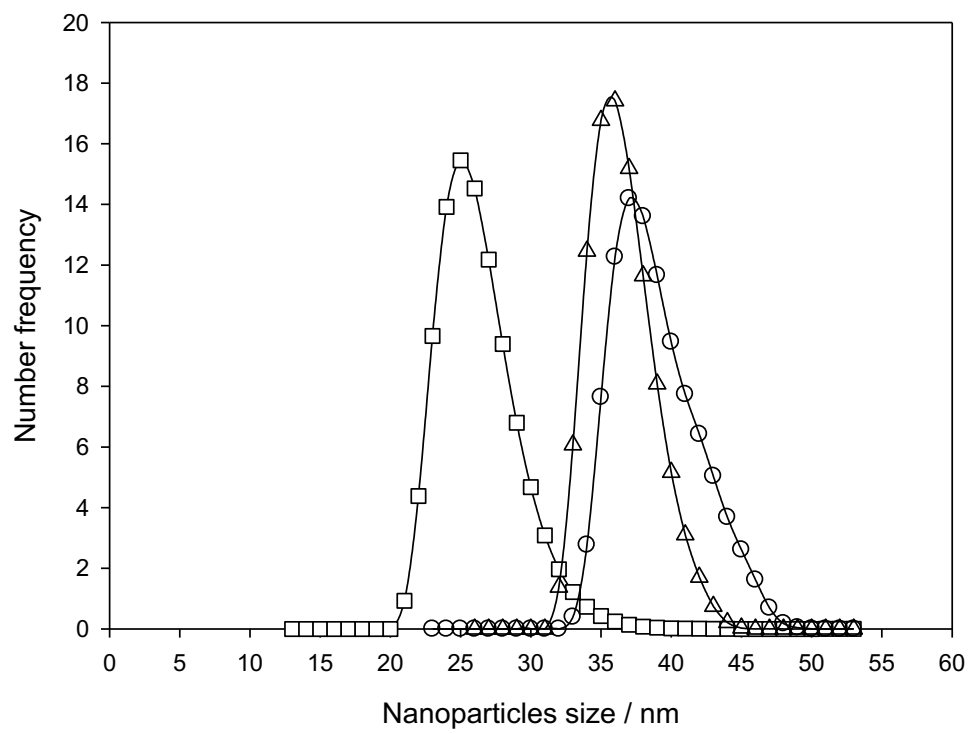

Figure 1: Size distributions of silica nanoparticles (squares), silica-titania core-shell nanoparticles (STCSNP) (circles) and silica-titania hollow nanoparticles (STHNP) (triangles).

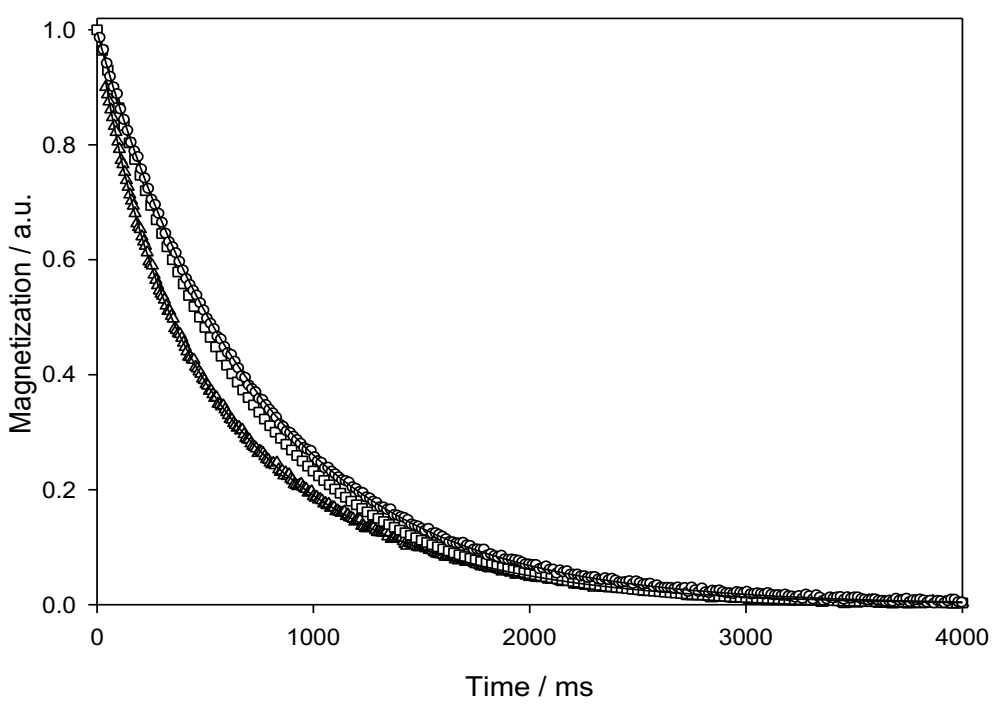

Figure 2: CPMG decay curves for silica nanoparticles (squares), silica-titania core-shell nanoparticles (STCSNP) (circles) and silica-titania hollow nanoparticles (STHNP) (triangles), at fixed volume fraction of 0.0024 . 

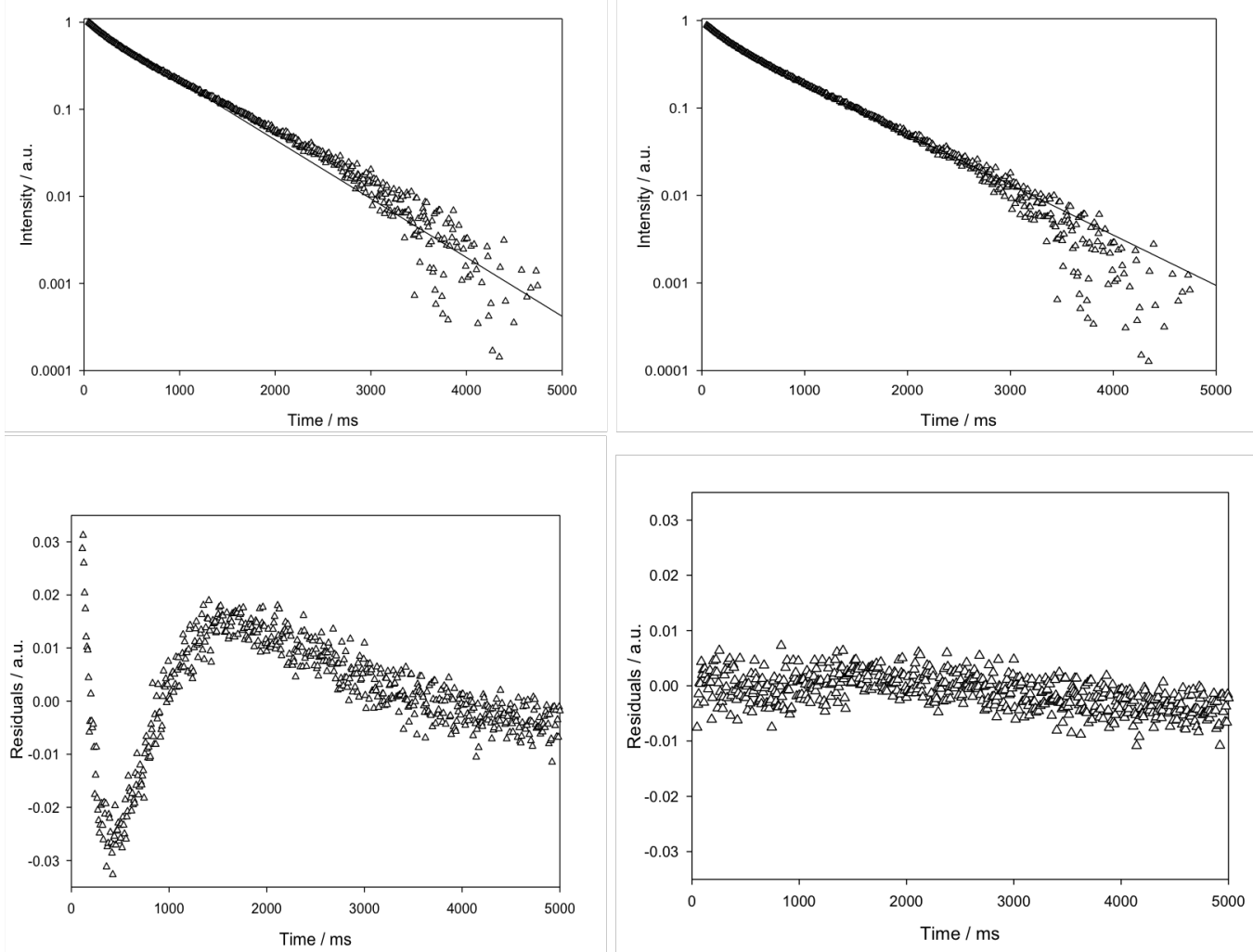

Figure 3: Relaxation decay curve for the hollow nanoparticles. The solid line through the experimental data (top panels) is the single exponential fit (left) and double exponential fit (right). The lower panels depict the residual plots - the difference between fit and the data - for the hollow nanoparticles calculated from the single exponential fit (left) and double exponential fit (right). 


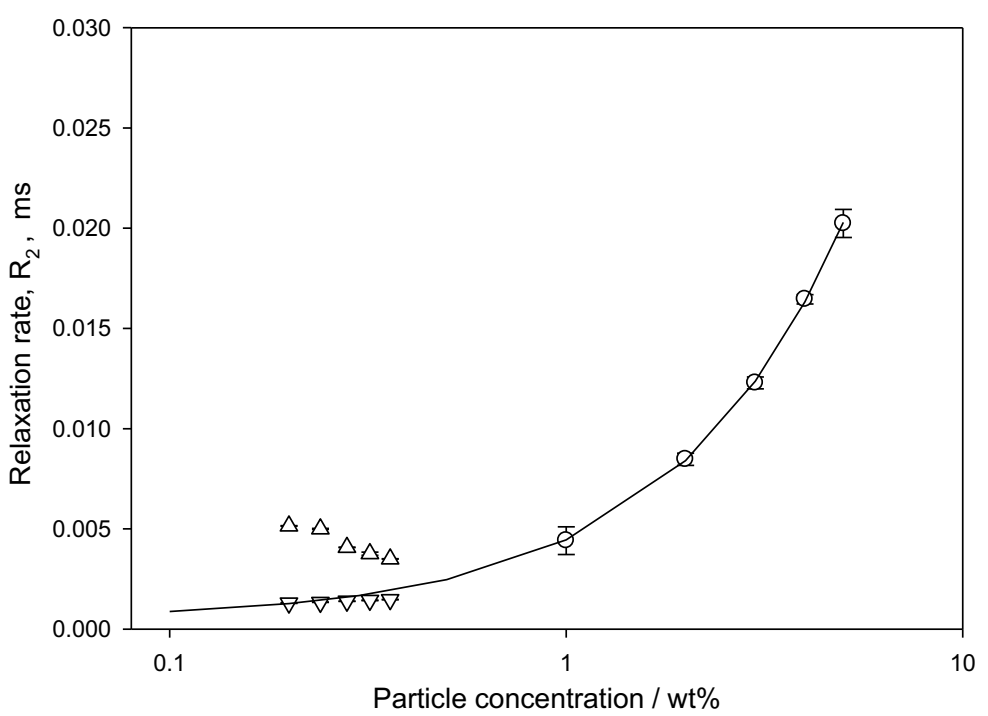

Figure 4: Variation in $\mathrm{R}_{2}$ components for hollow nanoparticles; (triangles up) fast rate and (triangles down) slow rate as a function of volume fraction. At higher volume fractions, only one rate is observed (circles). The solid line is a linear prediction of $\mathrm{R}_{2}$ on surface area.

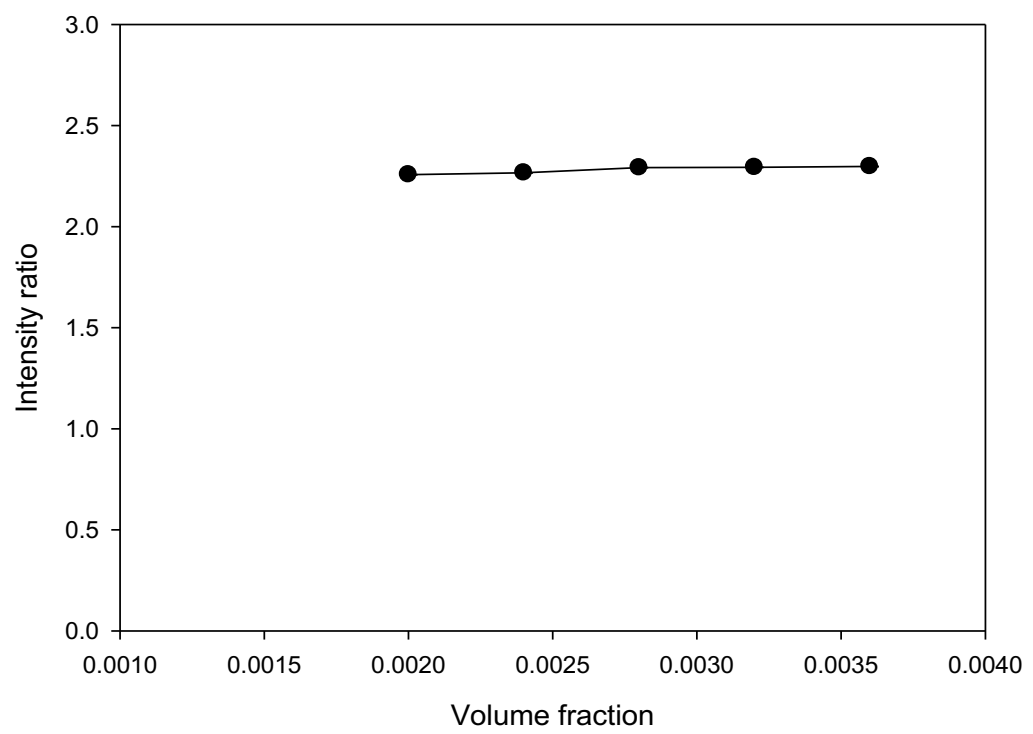

Figure 5: Intensity ratio (slow / fast) of the two relaxation components at low volume fraction for hollow particles. 


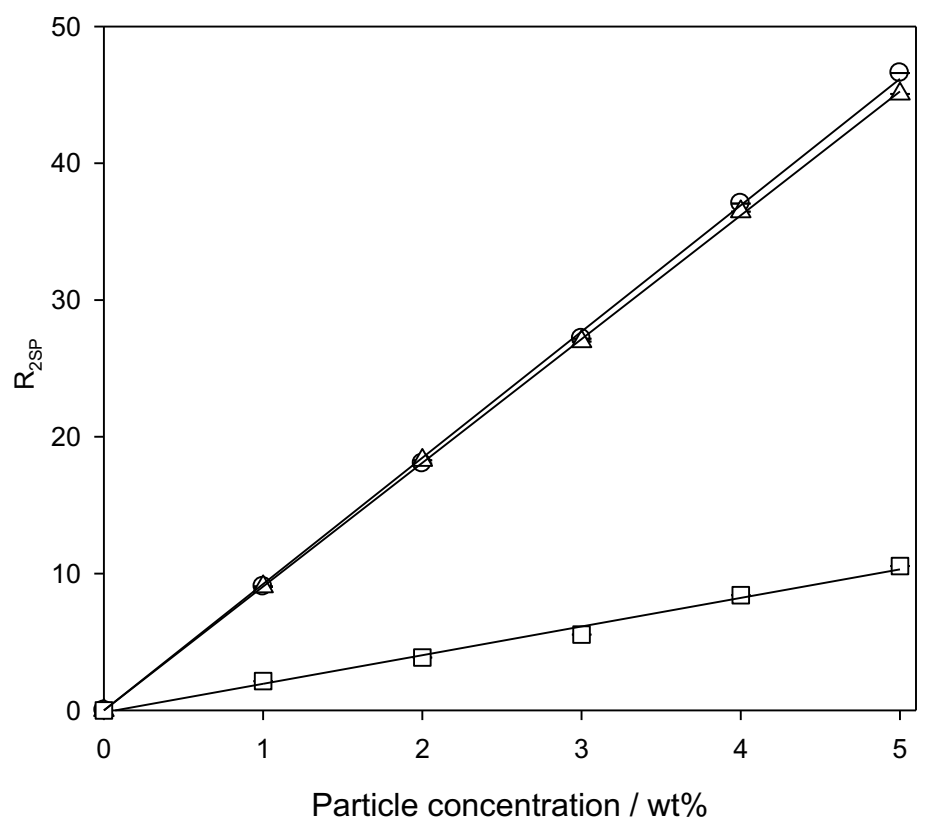

Figure 6: Relaxation rate $\left(\mathrm{R}_{2 \mathrm{SP}}\right)$ of silica nanoparticles (squares), silica-titania core-shell nanoparticles (STCSNP) (circles) and silica-titania hollow nanoparticles (STHNP) (triangles) as a function of particle concentration. 


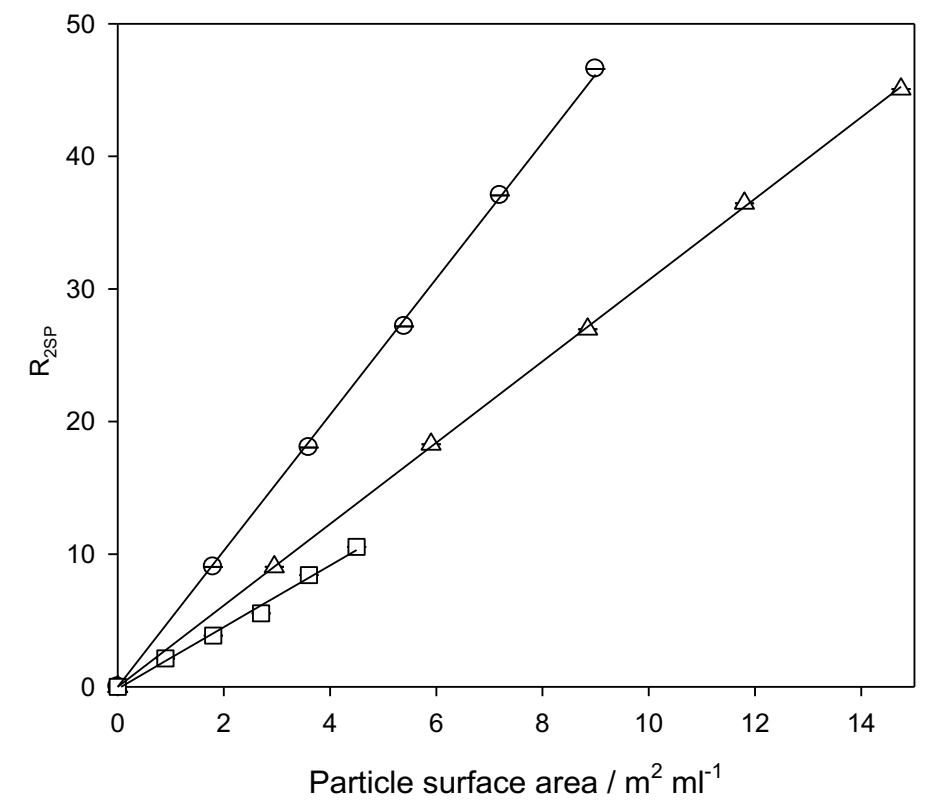

Figure 7: Relaxation rate $\left(\mathrm{R}_{2 \mathrm{SP}}\right)$ for silica nanoparticles (squares), silica-titania core-shell nanoparticles (STCSNP) (circles) and silica-titania hollow nanoparticles (STHNP) (triangles) as a function of particle surface area. The surface area used for three different colloidal particles were calculated from the average particle radius (measured from scanning-transmission electron microscopy) and the volume fraction. 
